\title{
Short-Term Site-Wide Maintenance Scheduling
}

\author{
Kwok-Yuen Cheung ${ }^{1}$, Chi-Wai Hui ${ }^{1,+}$, Haruo Sakamoto ${ }^{2}$, Kentaro Hirata ${ }^{2}$ \\ and Lionel O’Young ${ }^{3, *}$,
}

${ }^{1}$ Chemical Engineering Department, Hong Kong University of Science and Technology, Clear Water Bay, Hong Kong.

${ }^{2}$ Process Development and Design Lab., Process Systems Engineering and Production Technologies, MCC-Group Science \& Technology Research Center, Mitsubishi Chemical Corporation, 1, Toho-cho, Yokkaichi, Mie 510-8530, Japan.

${ }^{3}$ MC Research \& Innovation Center Inc., 444 Castro St., Mt. View, CA 94041, USA.

\footnotetext{
${ }^{+}$Author to whom all correspondence should be addressed. Email: kehui@ust.hk

*Current address: CWB Technology, Inc., 20311 Valley Blvd, Suite C, Walnut, CA91789, USA.
} 


\begin{abstract}
Preventive maintenance is essential for every chemical production site to prevent failure and accidents, however it upsets material and utility flows inside the site and also causes production loss. In order to minimize the loss, maintenance of each plant unit has to be carefully scheduled together with considerations on site-wide material and utility balances. This will involve both production and utility systems, and indeed is a very complicated problem. A scheduling strategy is then employed to handle the problem efficiently. It divides the scheduling into two steps, long-term and short-term. Long-term maintenance scheduling determines the combination of plant shutdown in a period of two to five years. Based upon the long-term schedule, a short-term maintenance scheduling optimizes the exact timing of plant shutdown, overhaul, inspection and startup within a maintenance period of four to ten weeks. Short-term maintenance scheduling involves pre-set utility and material demand profiles during a plant shutdown, overhaul and startup making it a very challenging task. In this paper, a multi-period mixed integer linear programming (MILP) model, a Site-Model, is proposed as an aid to optimize short-term site-wide maintenance schedule. A special formulation is also developed to deal with the pre-set utility and material demand profiles in the Site-Model.
\end{abstract}

Keywords: Site-Model, Site-Wide Optimization, Shutdown, Maintenance, Scheduling. 


\section{Introduction}

A chemical production site contains variety of production and utility plants. All of them require regular maintenance to enhance their reliability and to avoid emergency shutdowns. Nevertheless, plant maintenance is unfavorable on production suspension, huge capital and human resources involvement. It also affects the material and utility balances between the production and the utility units. An effective maintenance schedule should hence be able to balance all above factors and provide a feasible and economical result.

In this paper, a multi-period mixed integer linear programming (MILP) model, called Site-Model, is proposed for solving this type of maintenance scheduling problems. A site-model includes all the major material and utility balances of the production and utility plants as well as their interconnections and constraints.

To optimize a maintenance schedule, it requires tackling both long-term scheduling and short-term scheduling (Cheung and Hui, 2003). In a chemical production site, not every plant has to be maintained every year. There are always some plants being operated while others are down for maintenance. Due to the limitations on the availability of materials, utilities and skilled workers, the production site usually defines two to three maintenance periods in a year. Each period lasts for four to ten weeks. Production plants and utility facilities are then divided into groups and maintained separately in different maintenance periods. A long-term maintenance schedule provides grouping for maintenance plants and assigns a maintenance period to each group (rough maintenance timing) over a two to five year time span. It takes into account the basic material and utility balances during the whole scheduling time horizon, and to determine long-term production and inventory strategies as 
well as materials and utilities import/export policies.

Superimposed on the long-term maintenance schedule, short-term scheduling extracts the grouping results of maintenance plants from every maintenance period. It then further optimizes them by deciding the detail timing of the maintenance main operations (i.e. shutdown, overhaul, inspection and startup) of all the maintenance plants during a maintenance period (Figure 1). The main operations are normally completed by a relatively short period (four to ten weeks), however their utility and labor demands could be significantly fluctuated. The main objective of short-term scheduling is thus to guarantee a feasible operation, smooth utility and labor demands, and minimize production losses during the maintenance period.

Only a small number of literature addresses maintenance scheduling about chemical productions. Some of them focused on the short-term scheduling of multipurpose batch plants with integration of maintenance and production (Dedopoulos and Shah, 1995 and Pistikopoulos et al., 2001). Kim and Han (2001) have discussed maintenance scheduling of utility systems by assuming a fixed configuration of utility and production systems and fixed utility demands. Hui and Natori (1996) included maintenance feasibility as a consideration in a case study of utility system expansion. Tan and Kramer (1997) scheduled maintenance for chemical processes. So far, maintenance schedules for a chemical production complex including the interaction between production and utility systems have not been investigated in any of earlier works.

The idea of applying a site-model for long-term site-wide maintenance scheduling has 
previously been discussed by Cheung and Hui (2003). This paper will then focus on the short-term maintenance scheduling of a chemical production complex, which contains both production and utility plants.

\section{Problem Definitions}

A simple example site has been adopted from Hui (2000) to illustrate short-term scheduling in this paper (Figure 2). The example site consists of eight production plants (ETY, VCM, PVC, PE, PP, A, B and C). Five of them, ETY, VCM, PVC, PE and PP, connect sequentially and material flows are involved among them. The other three plants, A, B and C, are set up as groups of some batch processes. They can operate individually and only obtain utilities from the utility plants. Besides the production system, a utility plant that consists of two boilers (B1 and B2) and three turbines (T1, T2 and T3) are built for sustaining the utility supply.

Not all the processes and utility facilities need maintenance in every maintenance period. The long-term scheduling is therefore carried out to determine the plant grouping for each maintenance period. Based on that result, this paper's examples will only investigate the maintenance period that involves maintenance of plants ETY, VCM, PP, PE, PVC, boiler B2 and turbine T2. Other units are operated as usual at the period. The whole maintenance period lasts for a maximum of 31 days. Each day has three shifts (day, night and midnight). Prices and importation limits of electricity are different at each shift. Before a unit undergoes maintenance, a few days are required to shutdown all the process equipment. Overhaul is then carried out in the following days of weeks requiring intensive labor and heavy machinery involvements. Within the overhaul, inspection is carried out to ensure that 
the maintenance is done properly. After the overhaul, the plant requires a few days to resume (or startup) to normal operating conditions. Utility, material and manpower demands of the maintenance plants vary day by day according to pre-determined profiles during the shutdown, overhaul and startup. The variations can be treated as different stages in the shutdown, overhaul and startup (Figure 3), and these demand profiles are given as input for the optimization. The lengths of the shutdown and startup periods are fixed. The length of overhaul nevertheless could either be fixed at or extended from a lowest limit.

Given raw-material costs, product prices, inventory holding costs, fuel costs, electricity variable and fixed costs, and maintenance demand profiles, the site-model is objected to maximize the total site profit during the maintenance period by determining a suitable short-term maintenance schedule.

\section{Site-Model Definitions}

Maintenance scheduling involves material and utility balances, and other site-wide factors in a chemical production site. The problem scale is usually large and the problem is very complicated. This however is suitable for applying the technique of site-model.

A site-model is a general mathematical programming model that contains the major material and utility balances of both production and utility systems in a chemical production site. Some site-wide constraints, such as, seasonal production and utility demands, fuel and electricity supply contracts, can also be included. These constraints typically involve several well-defined sessions. For examples, seasonal demands vary according to the four seasons and electricity contract conditions change in normal operations and maintenance operations. 
Since the site-model is targeted on planning and scheduling problems, it is beneficial to formulate in a multi-period time frame (e.g. days, months or individual scenarios) to tackle that kind of constraints. Planning and scheduling usually are the preliminary step of other detail studies. As the solution accuracy will be increased through the subsequent detail studies, quick solution has priority over the accuracy in planning and scheduling. The site-model hence adopts linear constraints for providing efficient and sufficiently accurate solutions in such large-scale problems (Cheung et al., 2002). Those problems normally require huge computational efforts with nonlinear models. Furthermore, nonlinear models are easily stuck in local optimum. If it is necessary, binary variables can also be imposed into the model for selecting equipment options and determining equipment on/off.

As number of parameters, equations and variables in a site-model increases rapidly with number of plant units and time periods, effective model management is necessary for applying the technique. Variables in a site-model are defined by three indices, a plant name (e.g. ETY - ethylene plant), a material name (e.g. MP - medium pressure steam) and an alternative (e.g. GEN - generation). With this three-indices system, variable names are more meaningful and model information like equations and variable bounds can be easily tabulated in a relational database. It is very useful on managing the model, especially for large industrial scale models.

\section{Model Formulations}

Site-model formulations are arranged carefully in order to match up its definitions. Notations are defined as follows. 
Indices:

$\begin{array}{ll}p & \text { Plant or unit } \\ m & \text { Material (includes utilities and manpower) } \\ a & \text { Alternative (property of variable) } \\ k^{S D} & \text { Alternative to indicate shutdown stage } \\ k^{O H} & \text { Alternative to indicate overhaul stage } \\ k^{S U} & \text { Alternative to indicate startup stage } \\ t, t^{\prime}, t^{\prime} & \text { Time period (day) } \\ s & \text { Shift in a time period } \\ r & \text { Material or utility balance equation index }\end{array}$

Sets:

$P \quad$ Set of plants and units

M Set of materials (includes utilities and manpower)

A Set of alternatives (property of variable)

$K^{S D} \quad$ Set of alternatives that indicate shutdown stage, $K^{S D} \in A$

$K^{\mathrm{OH}} \quad$ Set of alternatives that indicate overhaul stage, $K^{\mathrm{OH}} \in A$

$K^{S U} \quad$ Set of alternatives that indicate startup stage, $K^{S U} \in A$

$T \quad$ Set of time periods (day)

$S \quad$ Set of time shifts

$R \quad$ Set of material and utility balance equation indices

$T^{S D} \quad$ Set of time periods that can be the first shutdown stage, $T^{S D} \in T$

$T^{S U} \quad$ Set of time periods that can be the first startup stage, $T^{S U} \in T$ 
Set of time periods that inspection can be executed, $T^{I S} \in T$

$P^{M A}$

Set of plants that require maintenance, $P^{M A} \in P$

Parameters:

$E_{r, p, m, a, t, s} \quad$ Coefficient corresponds to variable $F_{p, m, a, t, s}$ in balance equation $r$

$U_{p, m, a, t, s} \quad$ Upper bounds of variable $F_{p, m, a, t, s}$

$L_{p, m, a, t, s} \quad$ Lower bounds of variable $F_{p, m, a, t, s}$

$S L_{s} \quad$ Length of time shift $S$

$L_{p}^{S D} \quad$ Length of shutdown period for plant $p$

$L_{p} \quad$ Length of overhaul period for plant $p$

$L_{p}^{S U} \quad$ Length of startup period for plant $p$

$L_{p}^{I S} \quad$ Length of time interval between inspection and startup of plant $p$

$\operatorname{Dem}_{p, m, a, k}^{S D} \quad$ Plant $p$ material demand for material $m$ with alternative $a$ in shutdown stage $k^{S D}$

$\operatorname{Dem}_{p, m, a}^{\mathrm{OH}} \quad$ Plant $p$ material demand for material $m$ with alternative $a$ during overhaul

$\operatorname{Dem}_{p, m, a, k}^{S U} \quad$ Plant $p$ material demand for material $m$ with alternative $a$ in startup stage $k^{S U}$

$I I N V_{m} \quad$ Initial inventory level of material $m$

$C_{p, m, a, t, s} \quad$ Cost/price of material $m$ with alternative $a$ at plant $p$ in period $t$, shift $s$

ELFix Electricity importation fixed cost

As shown in Figure 3, plant maintenance has to follow the procedures.

Shutdown $\rightarrow$ Overhaul (with inspection inside) $\rightarrow$ Startup

If time spans of all maintenance procedures are fixed and provided, timing of the plant maintenance will only depend on the first day of the plant shutdown (Figure 4). Therefore, 
the model requires binary variables to indicate plants' first shutdown day within the planning horizon.

Binary variables:

$z_{p, t}^{S D} \quad$ Binary variable to indicate that first shutdown day of plant $p$ occurs at period $t, p \in P^{M A}$

Due to the material demands will vary during the maintenance, it is also necessary to identify which maintenance stage (stage inside shutdown, overhaul and startup) is undergoing. This can be achieved by implementing some $0 \sim 1$ continuous variables. By carefully formulating with the binary variable $z_{p, t}^{S D}$, the $0 \sim 1$ continuous variables will only have a value 0 or 1 .

$0 \sim 1$ continuous variables:

$y_{p, t}^{S U} \quad$ Variable to indicate that first startup day of plant $p$ occurs at period $t, p \in P^{M A}$ $x_{p, t, k^{S D}}^{S D} \quad$ Variable to indicate that shutdown stage $k^{S D}$ of plant $p$ occurs at period $t$, $p \in P^{M A}$

$x_{p, t, k}^{S U} \quad$ Variable to indicate that startup stage $k^{S U}$ of plant $p$ occurs at period $t, p \in P^{M A}$

$w_{p, t}^{\mathrm{OH}} \quad$ Variable to indicate that overhaul of plant $p$ occurs at period $t, p \in P^{M A}$

$w_{p, t}^{I S} \quad$ Variable to indicate that inspection of plant $p$ occurs at period $t, p \in P^{M A}$

Positive continuous variables:

$F_{p, m, a, t, s} \quad$ Flow rate/amount variable of material $m$ with alternative $a$ at plant $p$ in period $t$, shift $s$ 
$I N V_{m, t, s} \quad$ Variable represents inventory of material $m$ in period $t$ shift $s$ in the storage tank

$S T i n_{m, t, s} \quad$ Variable represents material $m$ flow rate into the storage tank in period $t$ shift $s$ STout $_{m, t, s} \quad$ Variable represents material $m$ flow rate out of the storage tank in period $t$ shift $S$

Continuous variables:

Profit $t_{t, s} \quad$ Total site profit in period $t$, shift $s$

Maintenance frequency equations:

Within the planning period, the plants are only required to maintain once.

$$
\begin{array}{ll}
\sum_{t} z_{p, t}^{S D}=1, & p \in P^{M A}, t \in T^{S D} \\
\sum_{t} y_{p, t}^{S U}=1, & p \in P^{M A}, t \in T^{S U}
\end{array}
$$

Shutdown and startup relation constraints:

Since all time spans of shutdown, overhaul and startup are fixed, the relation between the first shutdown day and the first startup day is defined as:

$$
\begin{array}{r}
y_{p, t}^{S U}-z_{p, t^{\prime}}^{S D}=0, \quad \text { where } t=t^{\prime}+L_{p}^{S D}+L_{p}^{O H}, \\
p \in P^{M A}, t \in T^{S U}, t^{\prime} \in T^{S D}
\end{array}
$$

$t$ and $t$ ' are pre-set according to the time spans of shutdown $\left(L_{p}^{S D}\right)$ and overhaul $\left(L_{p}^{O H}\right)$. For 
instance, if plant $p$ requires 3 days to shutdown and 15 days to overhaul, $\left(t, t^{\prime}\right)$ will equal to $(1,19 ; 2,20 ; \ldots$; etc.). Furthermore, not all the days in the planning period can be assigned as a starting day of shutdown and startup. As the previous case, the plant cannot be started up before day 19 because it needs at least 18 days for shutting down and overhauling. $t$ and $t^{\prime}$ are hence constrained in their corresponding feasible day sets $T^{S U}, T^{S D}$ respectively. Due to this equality, the continuous variable $y$ will also obtain the same value of binary variable $z$, 0 or 1.

\section{Shutdown and startup stages constraints:}

In order to determine the demand values according to the profiles, operation days of each shutdown and startup stages have to be determined. This can be achieved by following constraints.

$$
\begin{aligned}
& x_{p, t, k^{S D}}^{S D}+x_{p, t+1, \bar{k} k^{S D}+1}^{S D}+\cdots+x_{p, t^{\prime}, k^{S D}}^{S D}-L_{p}^{S D} \times z_{p, t}^{S D}=0, \\
& \text { where } \overline{k^{S D}}=\text { first component of } k^{S D} \text {, } \\
& \overline{\overline{k^{S D}}}=\overline{k^{S D}}+L_{p}^{S D}-1 \\
& t^{\prime}=t+L_{p}^{S D}-1 \\
& p \in P^{M A}, t \& t^{\prime} \in T^{S D}, k^{S D} \in K^{S D} \\
& x_{p, t, k^{S U}}^{S U}+x_{p, t+1, \overline{k^{S U}}+1}^{S U}+\cdots+x_{p, t^{\prime \prime}, \overline{k^{S U}}}^{S U}-L_{p}^{S U} \times y_{p, t}^{S U}=0 \\
& \text { where } \overline{k^{S U}}=\text { first component of } k^{S U} \text {, } \\
& \overline{\overline{k^{S U}}}=\overline{k^{S U}}+L_{p}^{S U}-1,
\end{aligned}
$$




$$
\begin{aligned}
& t^{\prime \prime}=t+L_{p}^{S U}-1 \\
& p \in P^{M A}, t \& t^{\prime \prime} \in T^{S U}, k^{S U} \in K^{S U}
\end{aligned}
$$

$k^{S D}$ and $k^{S U}$ indicate the order of days (stages) inside shutdown and startup respectively. The order of stage $k^{S D}$ (or $k^{S U}$ ) is increasing simultaneously with the order of time period $t$ in the variable $x$ sequence in Equation (4) (or 5), until the sequence contains exactly the number of $L_{p}^{S D}$ (or $L_{p}^{S U}$ ) components. As variables $y$ and $z$ only obtain 0 or 1 , the variables $x$ will then be forced to have the same value of $y$ or $z$ in the equations. For instance, plant $p$ begins its shutdown at day $10(t=10)$ and its shutdown lasts for 3 days $\left(L_{p}^{S D}=3\right)$. It implies that $z_{p, t=10}^{S D}=1, \quad x_{p, t=10, k^{S D}=1}^{S D}=1, \quad x_{p, t=11, k^{S D}=2}^{S D}=1$ and $x_{p, t=12, k^{S D}=3}^{S D}=1$ by according to Equation (4). Same idea is applied in Equation (5) for controlling plant startup.

\section{Overhauling constraints:}

Days in between shutdown and startup are recognized as overhaul interval and are constrained as follow:

$$
\begin{aligned}
& \sum_{t} z_{p, t}^{S D}+\sum_{t^{\prime}} y_{p, t^{\prime}}^{S U}-w_{p, t^{\prime \prime}}^{O H}=1, \quad \text { where } t+L_{p}^{S D}-1<t^{\prime \prime}<t^{\prime} \text { and }\left(t, t^{\prime}\right) \text { is pre-set as in (3), } \\
& \qquad \in P^{M A},\left(t, t^{\prime}\right) \in\left(T^{S D}, T^{S U}\right), t^{\prime} \in T \\
& \sum_{t} w_{p, t}^{O H}=L_{p}^{O H}, \\
& p \in P^{M A}, t \in T
\end{aligned}
$$

Equation (6) restricts the overhauling period to be between the last day of shutdown and the 
first day of startup exclusively. Equation (7) guarantees the length of overhauling.

Inspection constraints:

Inspection is normally fixed a few days before the plant startup.

$y_{p, t}^{S U}=w_{p, t^{\prime}}^{I S}$

where $t^{\prime}=t-L_{p}^{I S}$,

$$
p \in P^{M A}, t \in T^{S U}, t^{\prime} \in T^{I S}
$$

$L_{p}^{S D}$ is the number of day that inspection should be before the first startup day. Since the inspection will not be carried out during holidays or weekends, its feasible day set is defined in set $T^{I S}$.

With constraints on timing control, the material and utility balance equation can be formulated.

Material and utility balance equations for non-maintenance plants:

Based on the site-model three-indices naming system, a combination of $p, m$ and $a$ can represent a flow or amount variable of the material in the site. Material and utility balances are just the relations between some of these variables. Hence, each balance equation $r$ will match with a particular pre-defined set of $(p, m, a)$. When there is no maintenance, the plant will follow its original material and utility balance equations.

$$
\sum_{r, t, s}\left(E_{r,(p, m, a), t, s} \times F_{(p, m, a), t, s}\right)=0, \quad r \in R, p \notin P^{M A}, m \in M, a \in A, t \in T, s \in S
$$


Material and utility balance equation for maintenance plants:

During maintenance, the normal material and utility balances are interrupted by the demand profiles. There is no more degree of freedom for variable $F$. When a maintenance stage occurs $\left(k^{S D}, k^{S U}\right.$ or $\left.k^{\mathrm{OH}}\right), F$ has to equal to its corresponding profile value. Hence, the balance equation here is only keeping the profile valid rather than determining a balanced value of $F$. A special linear constraint is then proposed to tackle this situation.

$$
\begin{aligned}
& \sum_{r, t, s}\left(E_{r,(p, m, a), t, s} \times F_{(p, m, a), t, s}\right)+\sum_{r, t, s}\left(E_{r,\left(p, m, k^{S D}\right), t, s} \times y_{p, t, k^{S D}}^{S D}\right)+\sum_{r, t, s}\left(E_{r,\left(p, m, k^{S U}\right), t, s} \times y_{p, t, k^{S U}}^{S U}\right) \\
& +\sum_{r, t, s}\left(E_{r,\left(p, m, k^{O H}\right), t, s} \times w_{p, t}^{O H}\right)=0, \quad r \in R, p \in P^{M A}, m \in M, a \in A, \\
& k^{S D} \in K^{S D}, k^{S U} \in K^{S U}, k^{O H} \in K^{O H}, t \in T, s \in S
\end{aligned}
$$

By definition, $K^{S D}, K^{S U}$ and $K^{\mathrm{OH}}$ are elements of alternative set $A$. Thus, the pre-defined set $(p, m, a)$ can be classified into $(p, m, a),\left(p, m, k^{S D}\right),\left(p, m, k^{S U}\right)$ and $\left(p, m, k^{O H}\right)$ for normal operation, shutdown, startup and overhaul respectively. A single equation is then able to cope with all the normal and maintenance operations simultaneously. This can eliminate a huge number of disjunctive equations for handling different operation conditions, and can save a lot of computational effort on solving the model. However, the remaining problem for applying

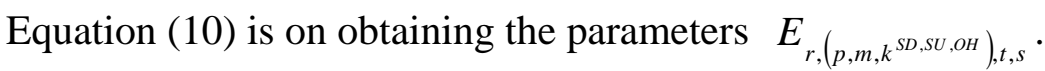

Since the maintenance demand profiles of different operation stages $k^{S D}, k^{S U}$ and $k^{O H}$ are known (i.e. variable pairs $F$ and $y$ (or $w$ ) are known), regression on Equation (10) can be used to determine parameters $E$ by substituting known pair of $F$ and $y$ ( $w$ for overhaul). Detail of 
the regression method has been discussed by Cheung et al. (2002).

Due to the complexity of the maintenance operations, variable bounds of $F$ are also separated into two groups.

Bounds for non-maintenance plants:

$$
\begin{array}{ll}
F_{p, m, a, t, s} \leq U_{p, m, a, t, s}, & p \notin P^{M A}, m \in M, a \in A, t \in T, s \in S \\
F_{p, m, a, t, s} \geq L_{p, m, a, t, s}, & p \notin P^{M A}, m \in M, a \in A, t \in T, s \in S
\end{array}
$$

Bounds for maintenance plants:

Unlike the non-maintenance plants, the variable bounds of the maintenance plants vary during the operations. This is because the demand profile values may violate the normal bounds. The following equations allow the bounds of the maintenance plant to be varied during and outside the maintenance period.

$$
\begin{aligned}
F_{p, m, a, t, s} \leq & U_{p, m, a, t, s}+\sum_{k^{S D}}\left(\left(\operatorname{Dem}_{p, m, a, k}^{S D}-U_{p, m, a, t, s}\right) \times y_{p, t, k}^{S D}\right) \\
& +\sum_{k^{S U}}\left(\left(\operatorname{Dem}_{p, m, a, k^{S U}}^{S U}-U_{p, m, a, t, s}\right) \times y_{p, t, k}^{S U}\right)+\left(\operatorname{Dem}_{p, m, a}^{O H}-U_{p, m, a, t, s}\right) \times w_{p, t}^{O H}, \\
& p \in P^{M A}, m \in M, a \in A, k^{S D} \in K^{S D}, \\
& k^{S U} \in K^{S U}, t \in T, s \in S \\
F_{p, m, a, t, s} \geq & L_{p, m, a, t, s}+\sum_{k^{S D}}\left(\left(\operatorname{Dem}_{p, m, a, k}^{S D}-L_{p, m, a, t, s}\right) \times y_{p, t, k}^{S D}\right) \\
& +\sum_{k^{S U}}\left(\left(\operatorname{Dem}_{p, m, a, k}^{S U}-L_{p, m, a, t, s}^{S D}\right) \times y_{p, t, k}^{S U}\right)+\left(\operatorname{Dem}_{p, m, a}^{O H}-L_{p, m, a, t, s}\right) \times w_{p, t}^{O H},
\end{aligned}
$$




$$
\begin{aligned}
& p \in P^{M A}, m \in M, a \in A, k^{S D} \in K^{S D}, \\
& k^{S U} \in K^{S U}, t \in T, s \in S
\end{aligned}
$$

Process operation and maintenance can be simulated by above equations and variables. Nevertheless, inventory is always kept to sustain downstream productions. It is necessary to model inventory strategy together with the maintenance schedule.

Inventory control constraints:

For the first shift of the whole planning period:

$$
\begin{gathered}
I N V_{m, \bar{t}, \bar{s}}=I I N V_{m}+S \operatorname{Tin}_{m, \bar{t}, \bar{s}} \times S L_{-s}-S T o u t_{m, \bar{t}, \bar{s}} \times S L_{-}^{-}, \\
\text {where } \overline{\bar{t}}=\text { first component of } t, \\
\bar{s}=\text { first component of } s, \\
m \in M, t \in T, s \in S
\end{gathered}
$$

For the first shift of day $t$ :

$$
\begin{array}{rl}
I N V_{m, t, \bar{s}}=I N V_{m, t-1, \bar{s}}+S \operatorname{Tin}_{m, t, \bar{s}} \times S L_{-s}-S T o u t_{m, t, \bar{s}} \times S L_{-\bar{s}}, & \\
\text { where } t & \neq \text { first component of } t, \\
\bar{s} & =\text { first component of } s, \\
& ==\text { last component of } s, \\
s & m \in M, t \in T, s \in S
\end{array}
$$

For other shifts:

$$
I N V_{m, t, s}=I N V_{m, t, s-1}+S \operatorname{Tin}_{m, t, s} \times S L_{s}-S T o u t_{m, t, s} \times S L_{s},
$$




$$
\begin{gathered}
\text { where } t \neq \text { first component of } t, \\
s \neq \text { first component of } s, \\
m \in M, t \in T, s \in S
\end{gathered}
$$

\section{Profit Calculations:}

To calculate the production site profit, following equation is applied:

$$
\begin{array}{r}
\text { profit }_{t, s}=\sum_{p, m, a}\left(F_{p, m, a, t, s} \times C_{p, m, a, t, s} \times S L_{s}\right), \\
\qquad p \in P, m \in M, a \in A, t \in T, s \in S
\end{array}
$$

\section{Objective function:}

The objective is to maximize the total-site profit for the whole planning period with consideration on electricity fixed cost.

$$
\max \left(\sum_{t, s} \text { profit }_{t, s}-\text { ELFix }\right), \quad t \in T, s \in S
$$

Equations (1) to (19) are integrated together to configure the site-model. The site-model is solved by using solver OSL (IBM, 1991) on the General Algebraic Modeling System (GAMS) (Brooke, 1992).

Details of the model including numerical data, constraints and equations are given at the web page pse.ust.hk.

5. Case Studies 


\subsection{Base Case}

Currently, plant maintenance is scheduled by experience and with some simple heuristics. The main concern of the scheduling is to guarantee feasible material and utility balances. Payment for skilled labor is one major concern in scheduling process. As in-house skilled labors are limited, external labors are hired to supplement the manpower shortage if necessary. It however also increases the salary expenditure a lot. In order to minimize the salary expenditure, plants will maintain separately to reduce the manpower requirement at a time. This definitely can be treated as a scheduling heuristic. Other heuristics can also be considered during the scheduling, such as the maintenance priority of particular plants/units. To maintain steam balance, the boilers are normally the last to shutdown and the first to be started up. With above simple heuristics, a maintenance schedule can be manually created as in Figure 5. This schedule is then verified using the site model to make sure of its feasibility. The results of this case will be treated as a reference and will be compared with other cases.

\subsection{Case 1}

Figure 6 shows an optimum maintenance schedule generated using the site model directly. The model optimized the overall profit taking into account of the variation of electricity prices given in current electricity contract and other site-wide constraints.

Compared with the base case, this schedule increases the total labor cost. Since most of the plants are maintained in the early part of the maintenance period, increased the requirement of external skilled workers. Electricity cost has also increased due to production increase. In return, the overall profit increases, mainly due to the increase of PE production. The PE plant is shut down together with the ETY plant that reduces the need from ETY inventory 
allowing the downstream processes of ETY to increase production during and after the ETY plant shutdown. The PE production rates in the Base Case and Case 1 are shown in Figure 7.

\subsection{Case 2}

Assuming that variation of current electricity contract is allowed providing an additional degree of freedom in the optimization. Electricity cost in a long-term purchasing contract is normally divided into two parts; fixed cost and variable cost. The fixed cost of electricity depends mainly on the amount of peak importation that is normally happen during the main utility facilities shutdown. The variable cost is calculated based upon the overall electricity consumption. In this study, the current electricity contract allows maximum importation of 45MW during the shutdown period. The optimum schedule with consideration of renewing the electricity contract is shown in Figure 8. Peak consumption of electricity is reduced to 28MW resulting a 43 million Yen decrease in electricity cost. Since the production rate remains high compared with the Base Case, the overall electricity price is still 2 million Yen higher than the Base Case. Electricity importation levels for Case 1 \& 2 are shown in Figure 9.

Decreasing the electricity importation in fact constrains the production in a certain extent. In order to minimize the adverse effects by this additional constraint, the least profitable product C has to sacrifice. The overall production rate of product $\mathrm{C}$ is reduced a lot from case 1 to case 2, especially at the end of the maintenance period. The change on the plant $\mathrm{C}$ can also indicate the importance of the site-wide considerations. Since plant $\mathrm{C}$ is a non-maintenance plant, its operation normally is not accounted in traditional scheduling approach. However, 
the site-model shows that obtaining the optimum schedule requires suitable collaborations of all maintenance and non-maintenance plants/units. Figure 10 illustrates the production rates of plant $\mathrm{C}$ in case 1 and case 2.

\subsection{Case 3}

During plant shutdown and startup, process equipment has to follow particular procedures. Their time spans are usually not be able to change. On the other hand, equipment can be idle even finishing the overhaul. The overhauling interval can therefore be extended based on a minimum. In previous cases, the length of the overhauling interval is fixed. In Case 3, the length of the overhaul is allowed to change providing an additional degree of freedom for increasing profit. To achieve this, parts of the model are reformulated.

\section{Shutdown and startup relation constraints:}

Since the overhauling interval is not fixed, the starting time of plant startup cannot be determined by the shutdown time solely. The $0 \sim 1$ continuous variable $y_{p, t}^{S U}$ has to be converted into a binary variable to illustrate the startup time independently. Parameter $L_{p}^{O H}$ is also changed to refer the minimum overhauling span.

Following constraint substitutes Equation (3). It ensures that the startup will begin only after the minimum overhauling span.

$$
\begin{array}{r}
y_{p, t}^{S U}-z_{p, t^{\prime}}^{S D} \leq 0, \quad \text { where } t \geq t^{\prime}+L_{p}^{S D}+L_{p}^{O H}, \\
\quad p \in P^{M A}, t \in T^{S U}, t^{\prime} \in T^{S D}
\end{array}
$$


Overhauling constraints (6) and (7) are modified as below.

$$
\begin{aligned}
& \sum_{t} z_{p, t}^{S D}+\sum_{t^{\prime}} y_{p, t^{\prime}}^{S U}-w_{p, t^{\prime \prime}}^{O H}=1, \quad \text { where } t+L_{p}^{S D}-1<t^{\prime \prime}<t^{\prime} \text { and }\left(t, t^{\prime}\right) \text { is pre-set as in (20), } \\
& p \in P^{M A},\left(t, t^{\prime}\right) \in\left(T^{S D}, T^{S U}\right), t^{\prime \prime} \in T \\
& \sum_{t} w_{p, t}^{O H} \geq L_{p}^{O H}, \\
& p \in P^{M A}, t \in T
\end{aligned}
$$

Result of case 3 is shown in Figure 11. The PP plant has extended its overhauling interval from 11 days to 14 days. PP is the least profitable product while comparing with other maintenance processes. Extending the PP plant maintenance allows utilities and materials to be better utilized for other productions resulting a slightly better profit. A comparison of the cases is given in Table 1.

\section{Conclusions}

A MILP model, Site-Model, is evaluated in this paper for tackling a short-term site-wide maintenance scheduling problem. Demands profiles are applied for all materials and utilities requires for plants during the whole maintenance period. The number of binary variables is minimized by carefully formulating the decision variables for plant shutdown, overhaul, inspection and startup. The case studies demonstrated the importance of considering utilities, materials and inventories, as well as variation of utility contracts simultaneously while scheduling the maintenance. A good maintenance schedule should not only guarantee feasibility, but also maximize the overall profitability. 


\section{Reference}

Brooke, A., Kendrick, D., and Meeraus, A. (1992). GAMS - A User's Guide (Release 2.25). San Francisco, CA: The Scientific Press.

Cheung K.Y., Li, W.K., Hui, C.W., Sakamoto, H., Hirata, K., and O’Young, L. (2002). Universal Plant Model for Production Planning. Proceeding of the 5th Conference of Process Integration Modelling and Optimisation for Energy Saving and Pollution Reduction (PRES’02), Prague.

Cheung, K.Y. and Hui, C.W. (2003). Total-Site Scheduling for Better Energy Utilization. Journal of Cleaner Production. In press.

Dedopoulos, I.T. and Shah, N. (1995). Optimal Short-term Scheduling of Maintenance and Production for Multipurpose Plants. Industrial and Engineering Chemistry Research, 34, 192-201.

Hui, C.W. (2000). Determining Marginal Values of Intermediate Materials and Utilities Using a Site Model, Computers and Chemical Engineering, 24, 1023-1029.

Hui, C.W. and Natori, Y. (1996). An Industrial Application Using Mixed-Integer Programming Technique: A Multi-Period Utility System Model, Computers and Chemical Engineering, 20, S1577-S1582.

IBM. (1991). OSL (Optimization Subroutine Library) Guide and Reference (release 2). NY: 
Kingston.

Kim, J.H. and Han, C. (2001). Short-term Multiperiod Optimal Planning of Utility Systems Using Heuristics and Dynamic Programming. Industrial and Engineering Chemistry Research, 40, 1928-1938.

Pistikopoulos, E.N., Vassiliadis, C.G., Arvela, J., and Papageorgiou, L.G. (2001). Interactions of Maintenance and Production Planning for Multipurpose Process Plants — A System Effectiveness Approach. Industrial and Engineering Chemistry Research, 40, 3195-3207.

Tan, J.S. and Kramer, M.A. (1997) A General Framework for Preventive Maintenance Optimization in Chemical Process Operations. Computers and Chemical Engineering, 21 (12), 1451-1469. 


\section{Acknowledgements}

The authors would like to acknowledge financial support from the Research Grants Council of Hong Kong (Grant No. 6014/99P), and financial and technical supports from Mitsubishi Chemical Corporation, Yokkaichi Plant. 
List of Figures:

Figure 1. Connection between long-term and short-term scheduling

Figure 2. Sample site-model

Figure 3. Example of maintenance demand profile

Figure 4. Maintenance procedures timing relations

Figure 5. Schedule of base case

Figure 6. Schedule of case 1

Figure 7. PE production rates of base case and case 1

Figure 8. Schedule of case 2

Figure 9. Electricity importation of case 1 and 2

Figure 10 Plant C production rates of case 1 and 2

Figure 11. Schedule of case 3 
List of Tables:

Table 1. Results comparisons 


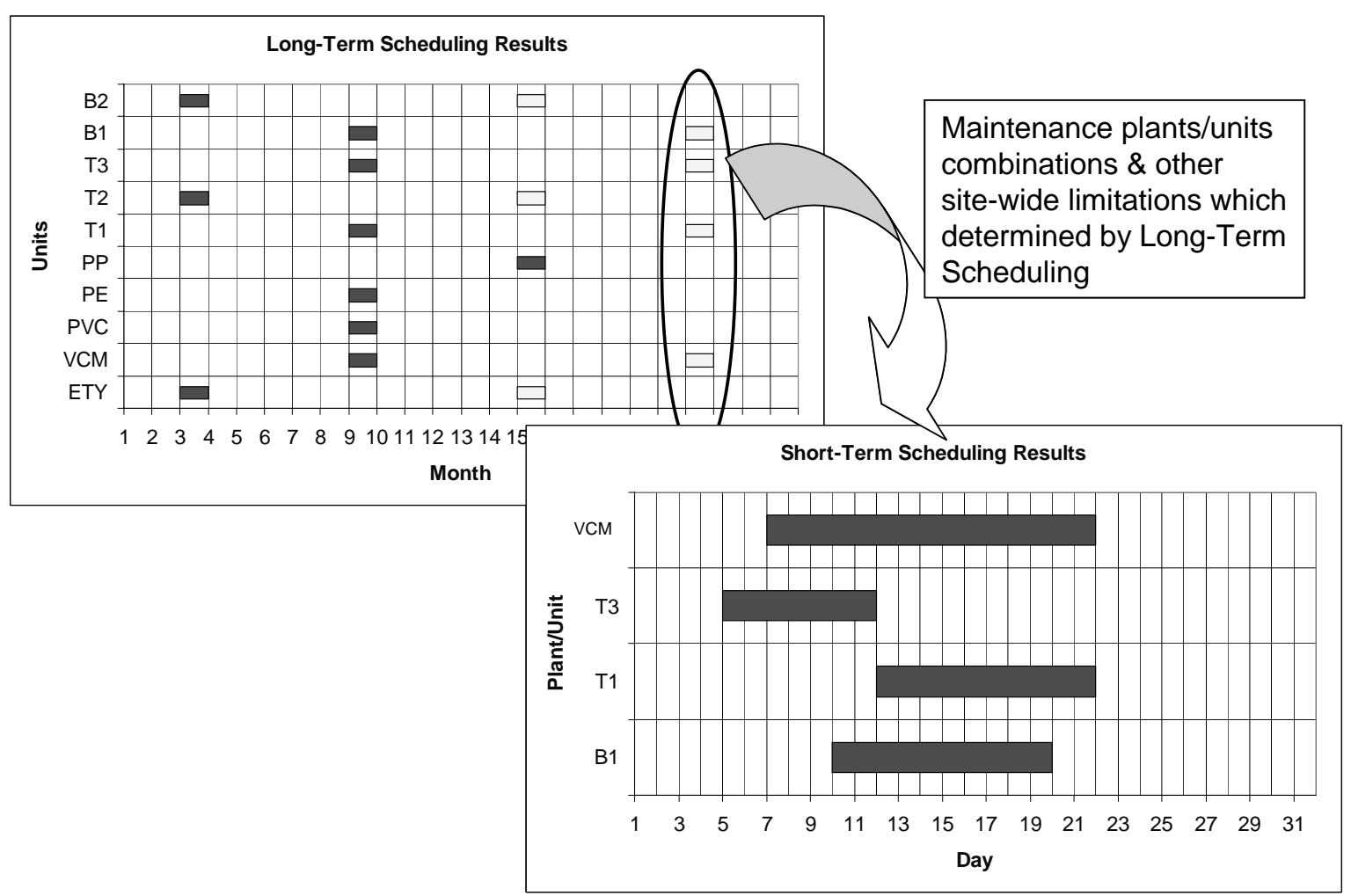

Figure 1 


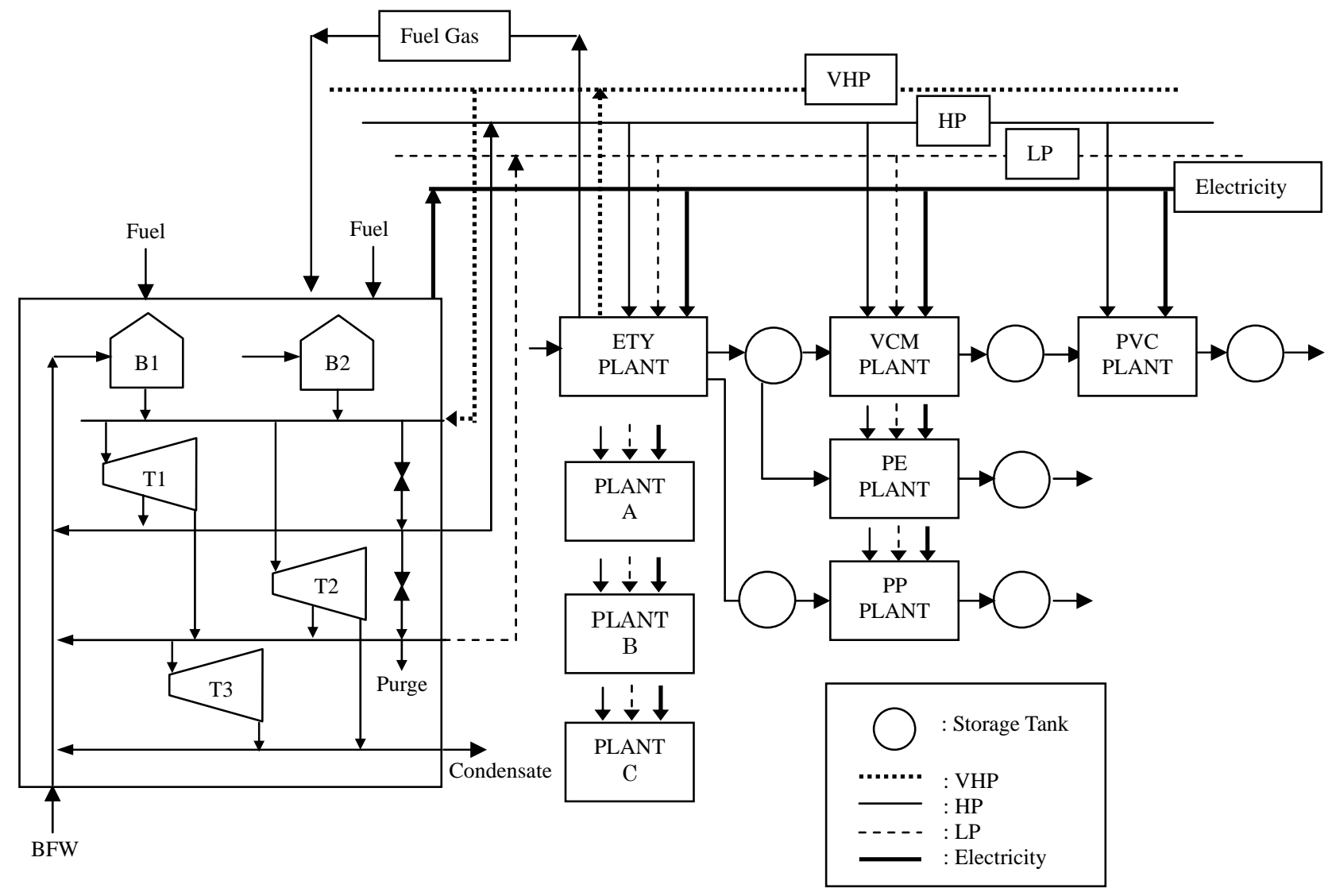

Figure 2 


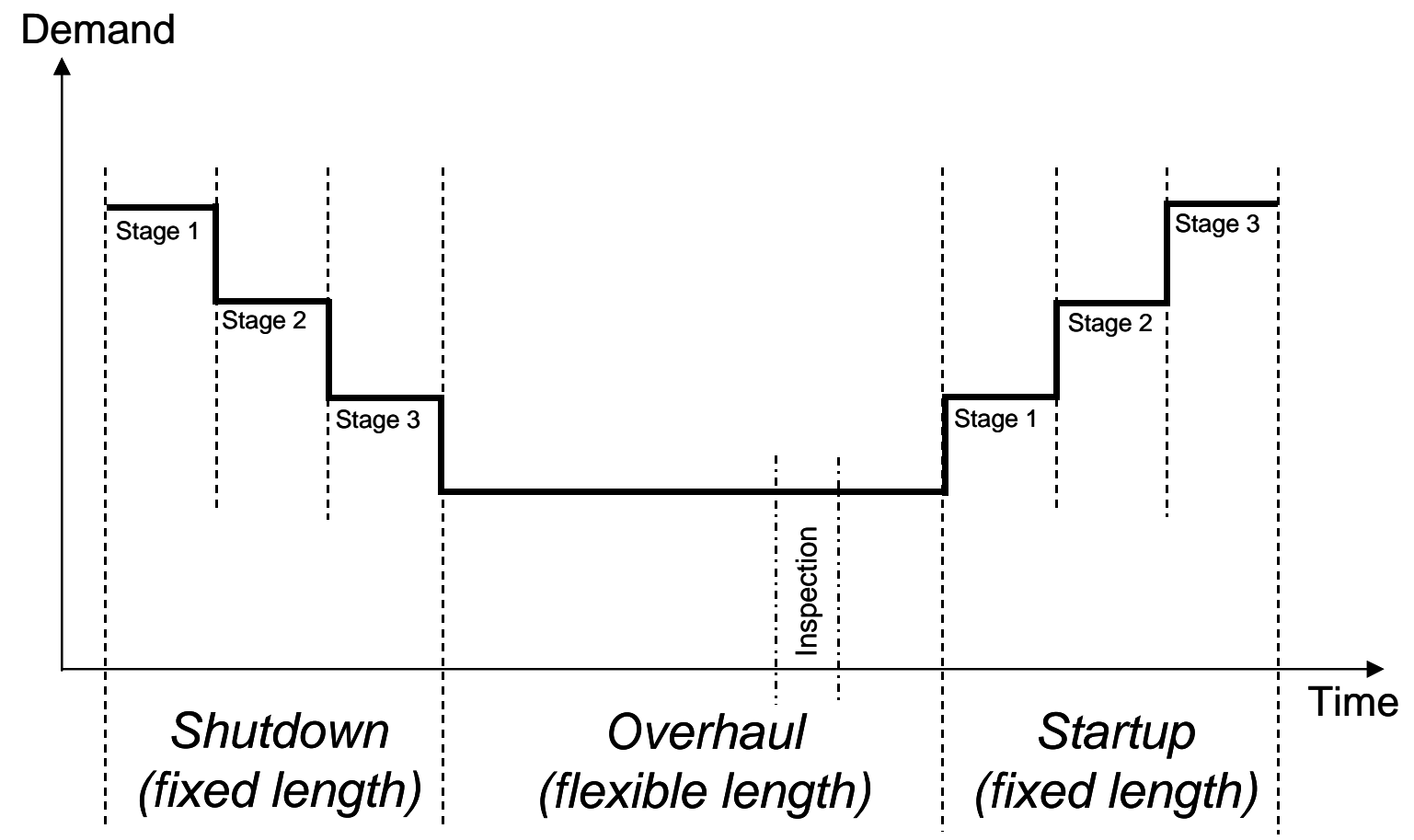

Figure 3 


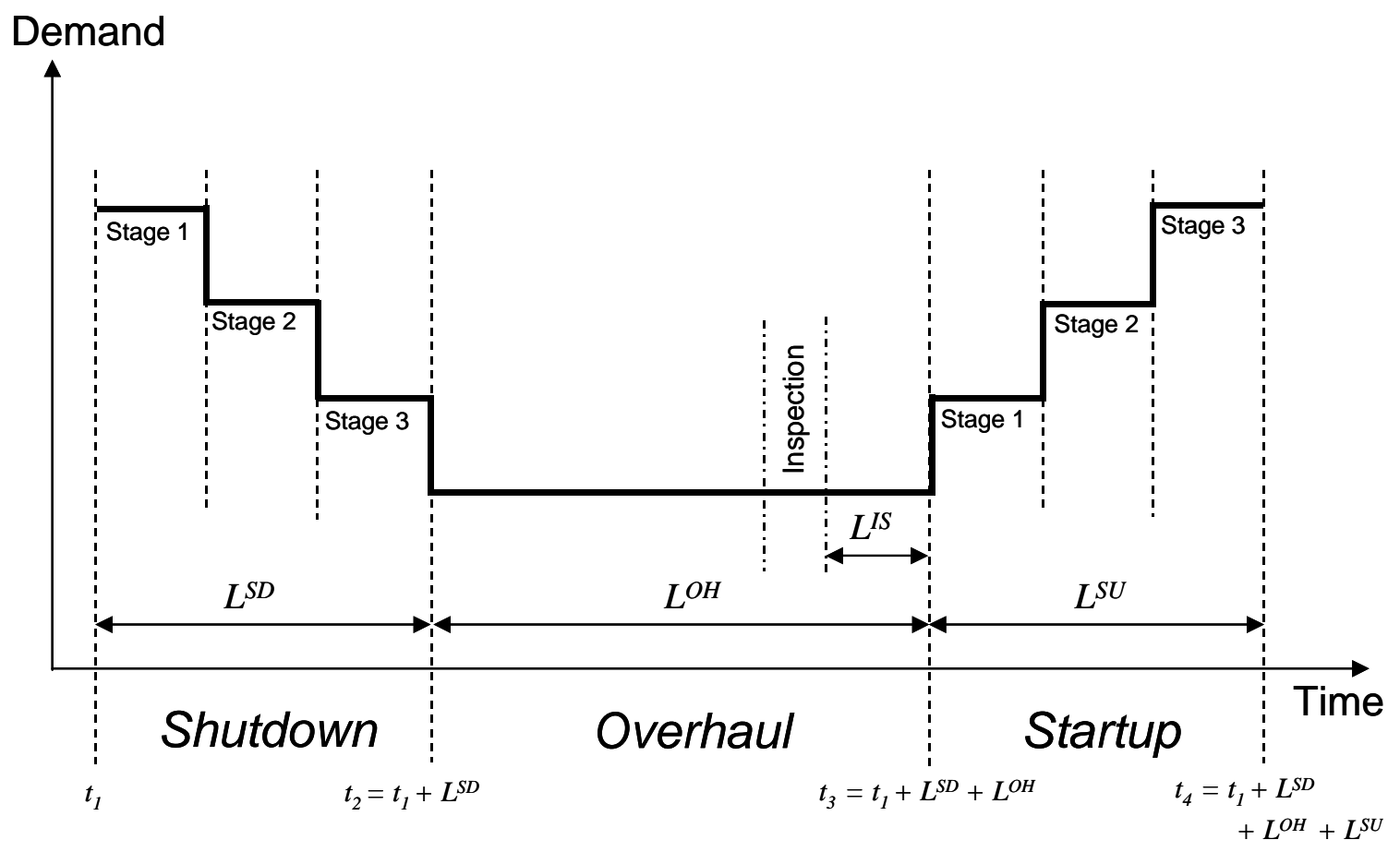

Figure 4 


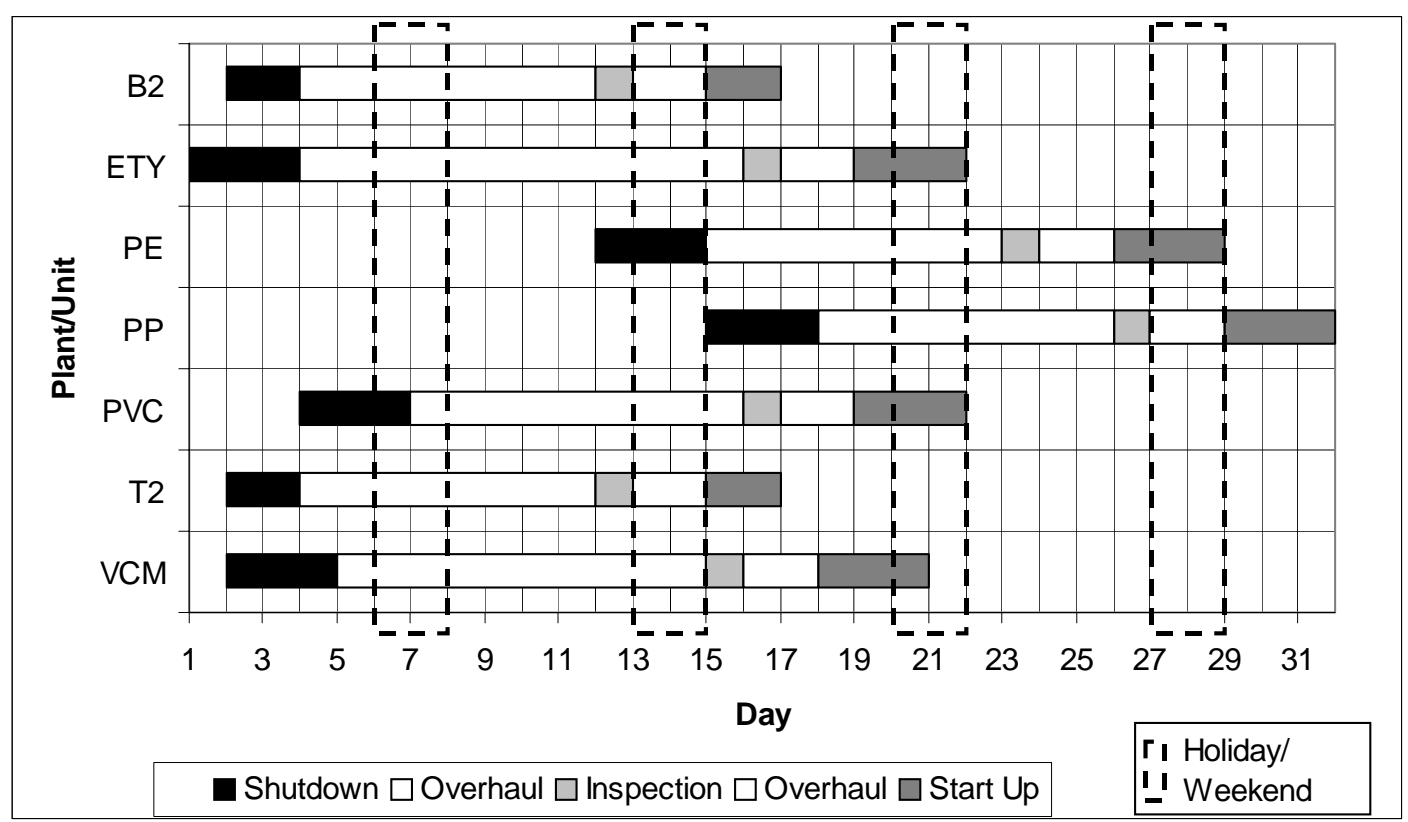

Figure 5 


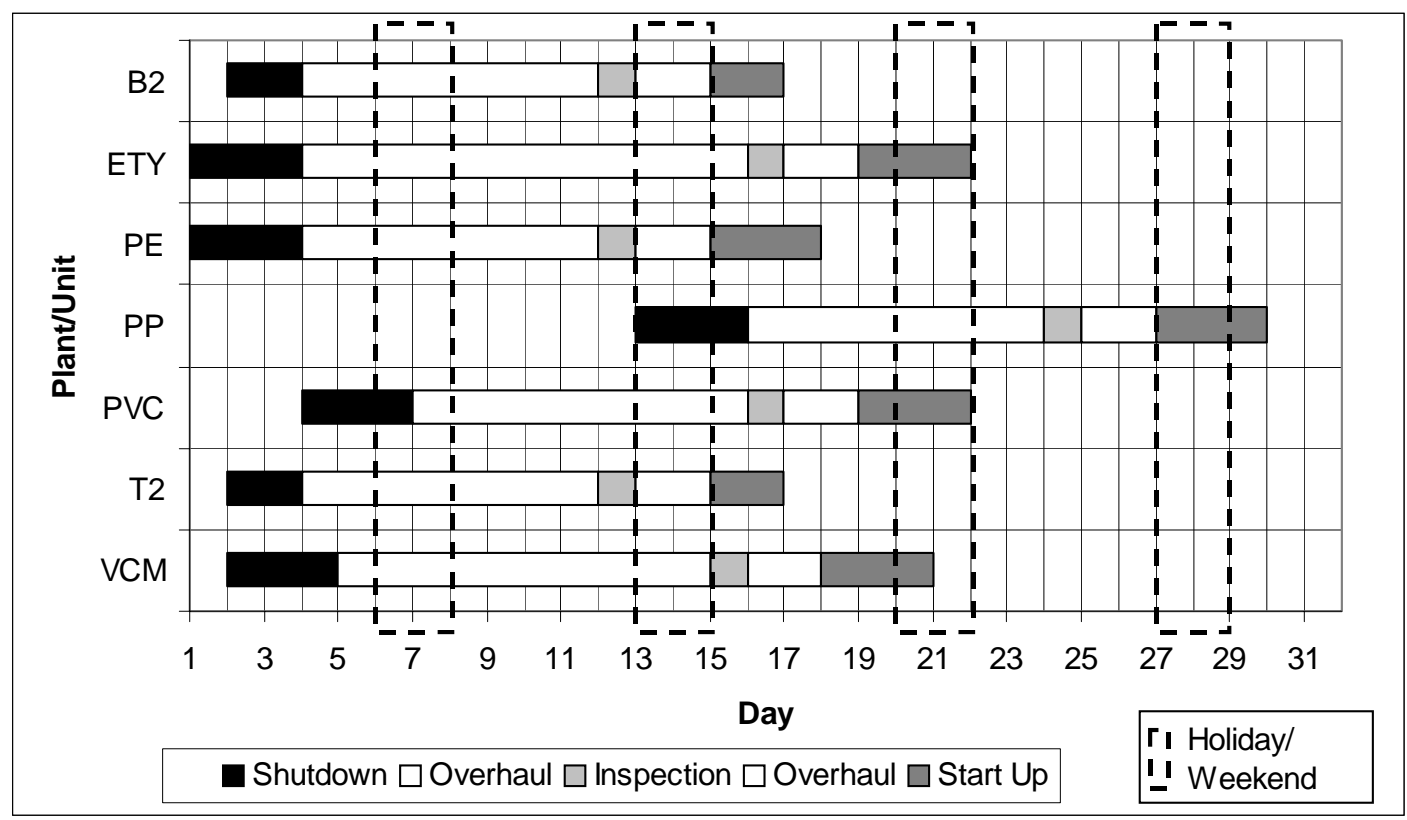

Figure 6 


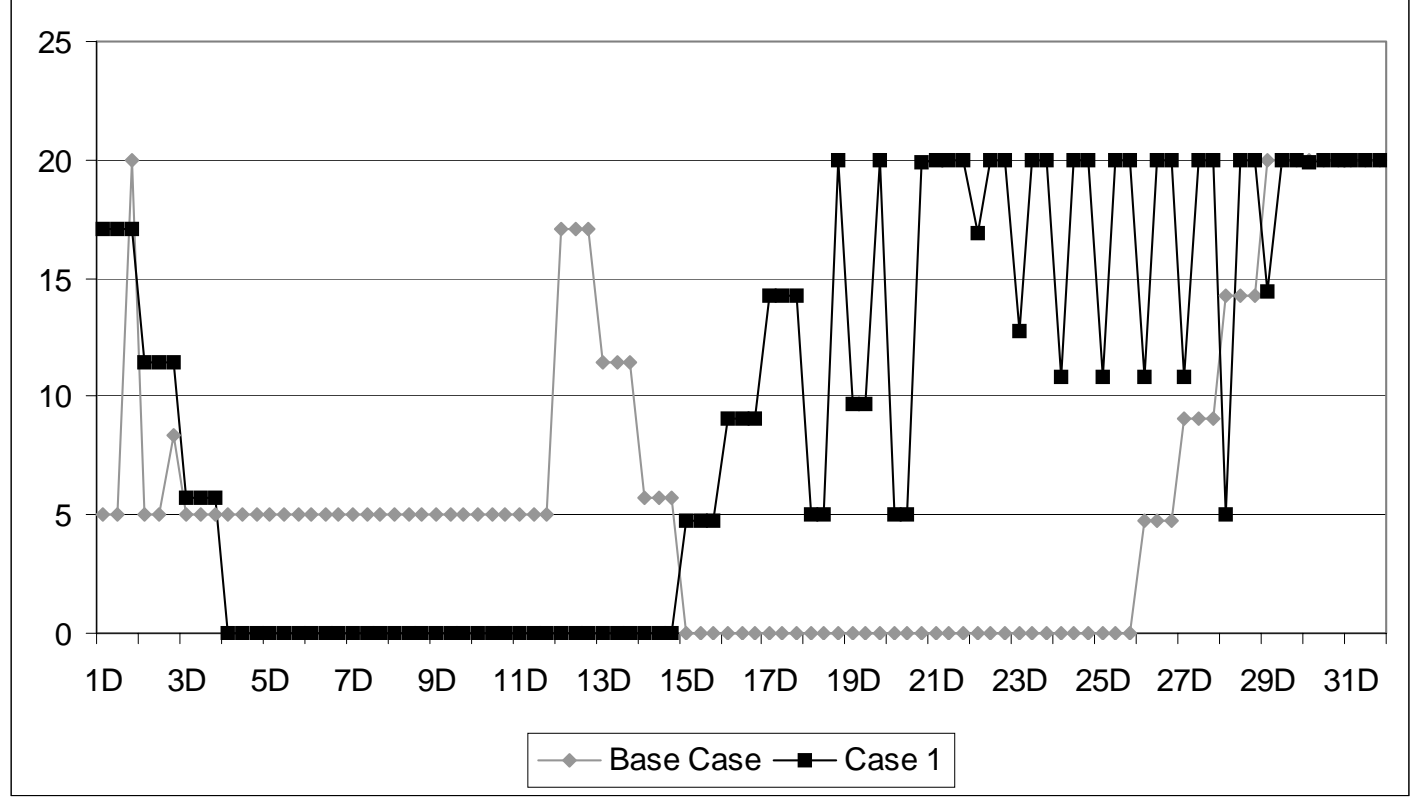

Figure 7 


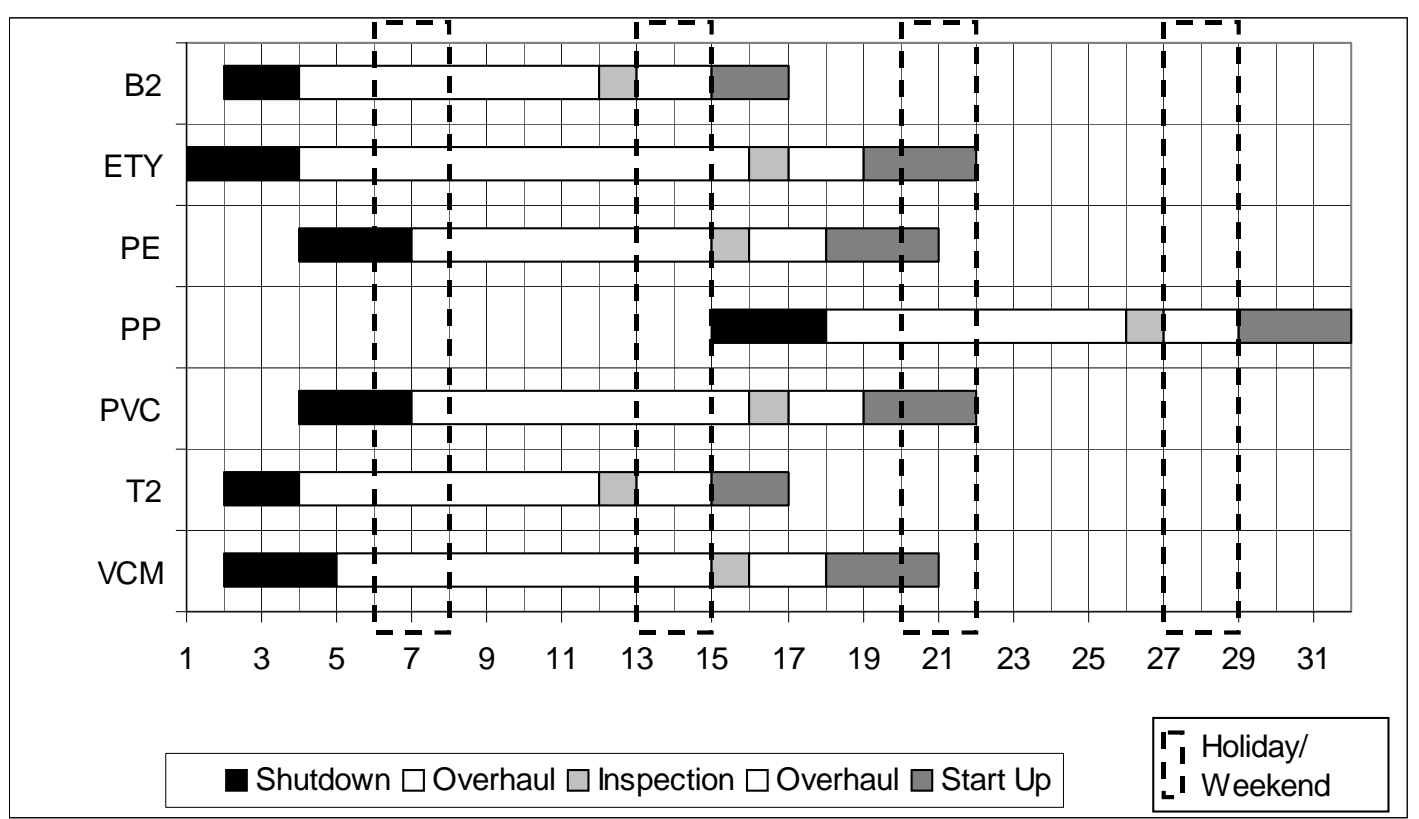

Figure 8 


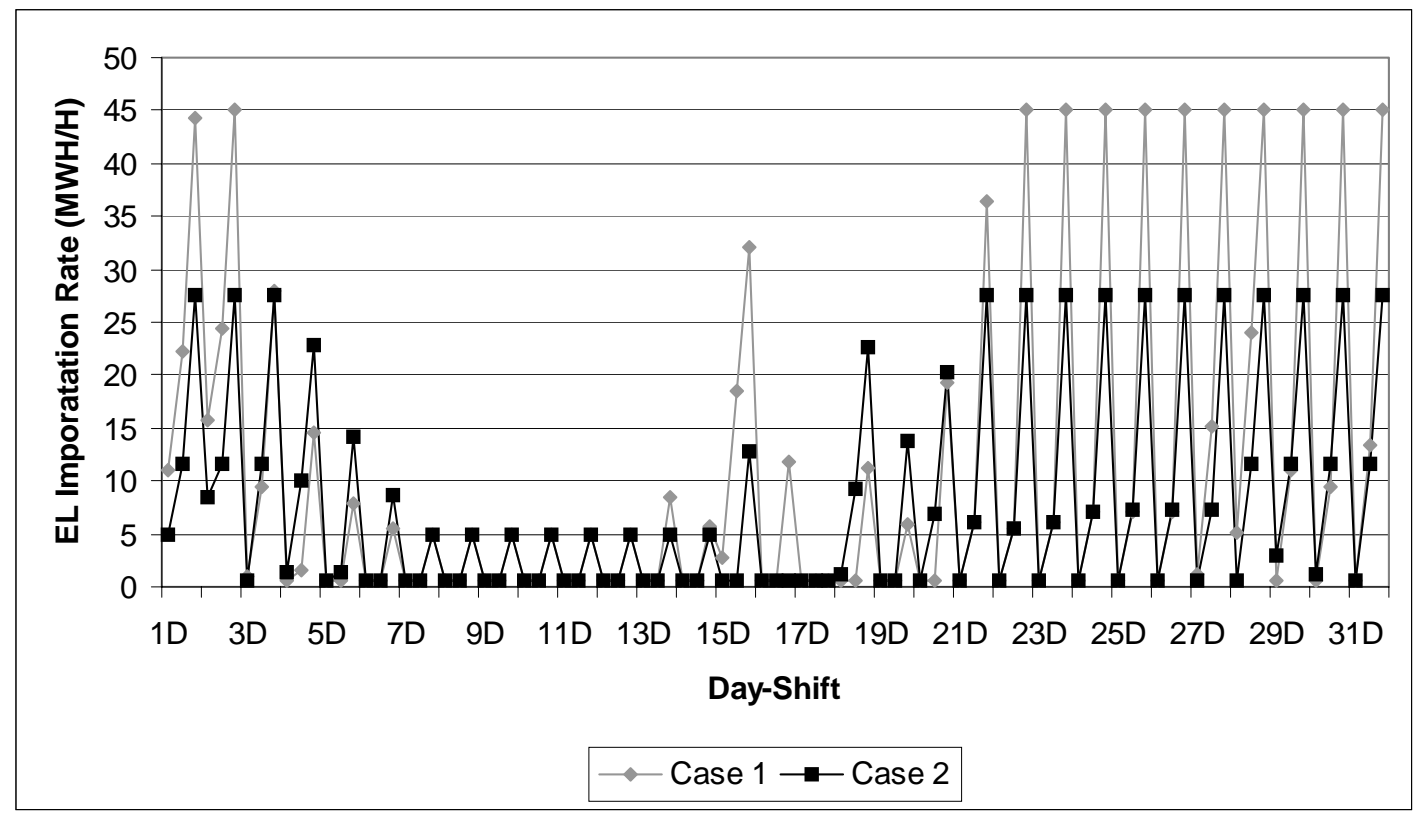

Figure 9 


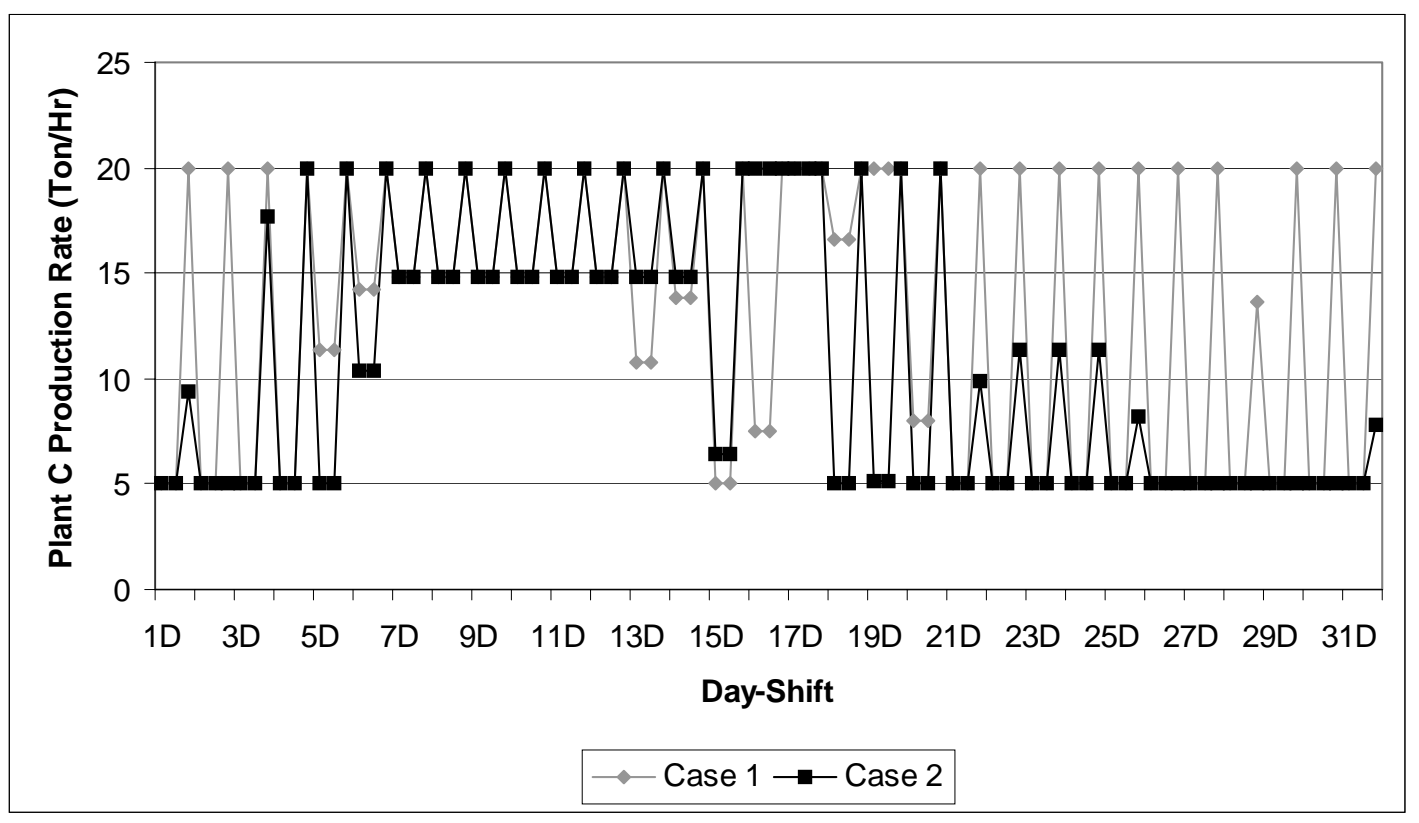

Figure 10 


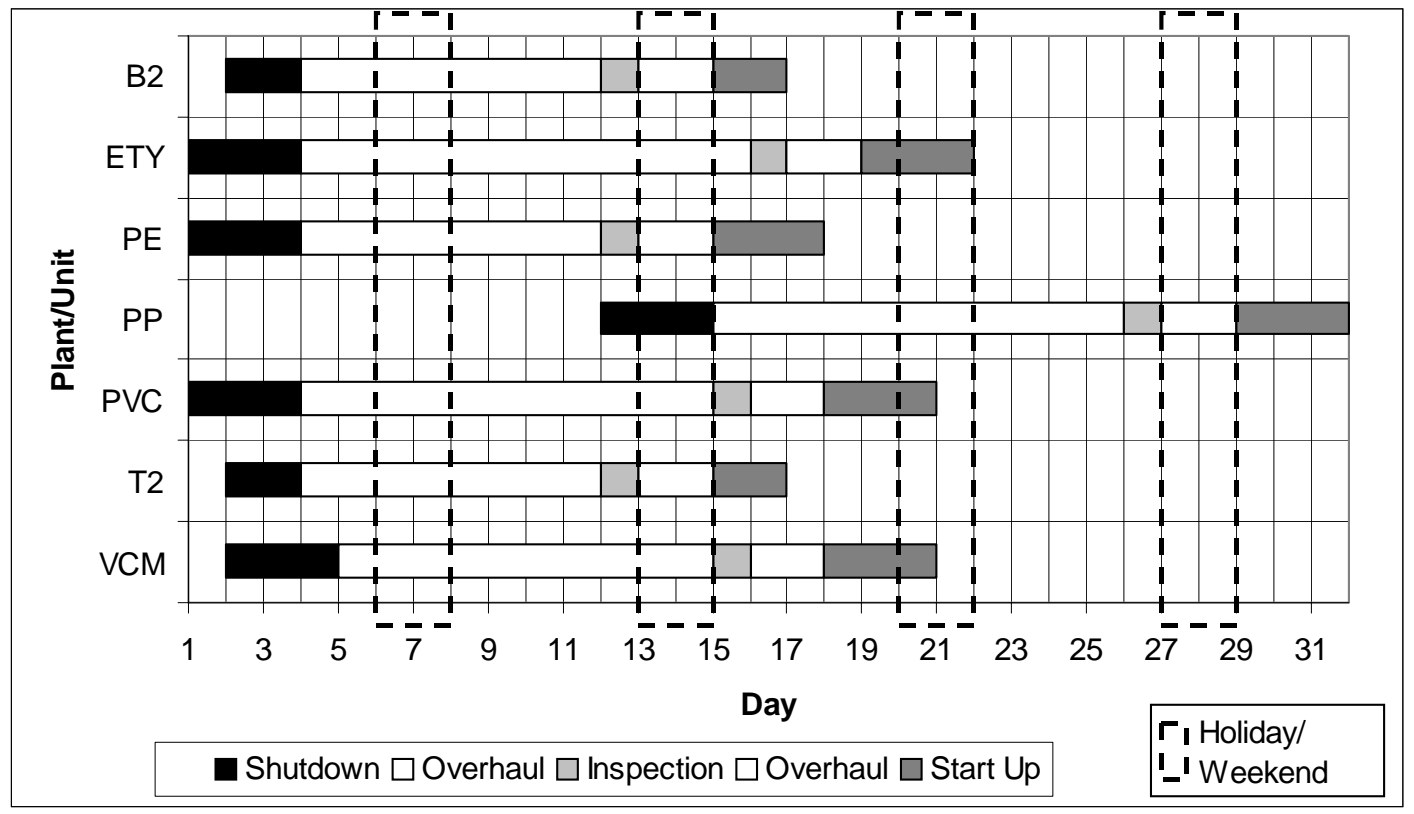

Figure 11 
Table 1

\begin{tabular}{lllll}
\hline Cost \& Profit & Base Case & Case 1 & Case 2 & Case 3 \\
$\begin{array}{llll}\text { (Million Yen) } \\
\text { Product Revenue }\end{array}$ & 2331 & 2707 & 2630 & 2600 \\
Labor Cost & -18 & -19 & -19 & -21 \\
Production Cost & -1175 & -1504 & -1411 & -1377 \\
Total Elect. Cost & -65 & -110 & -67 & -64 \\
Total Profit & 1137 & 1183 & 1200 & 1202 \\
\hline
\end{tabular}

